\title{
DISABILITIES OF THE ELBOW JOINT
}

\author{
W. E. TUCKER, C.V.O., F.R.C.S.
}

The Clinic, 71 Park Street, London W1Y 3HB.

The elbow joint is a ginglymus composite joint consisting of:

(a) the ulnar-humeral joint

(b) the radio-humeral joint

(c) the superior radio-ulnar joint; Fig. 1

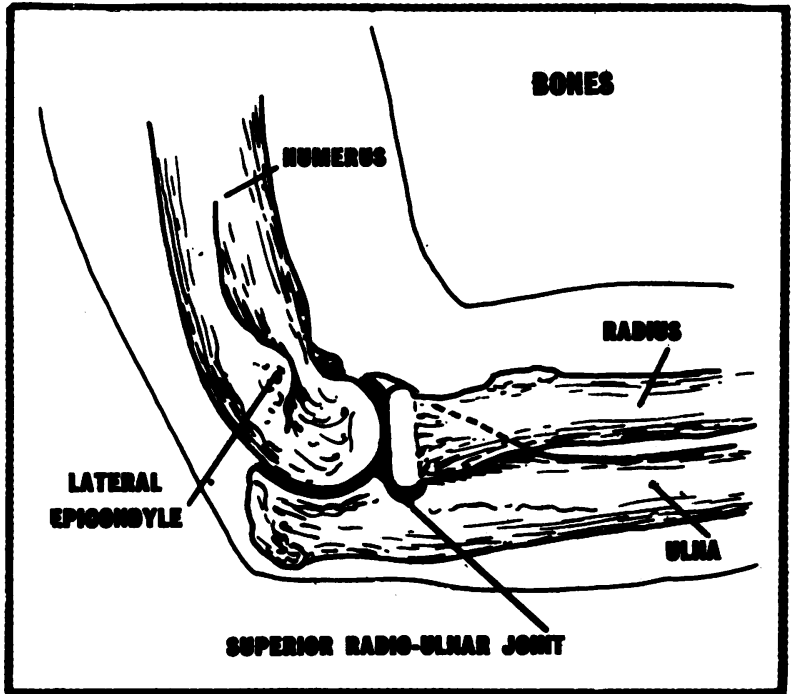

Fig. 1 This shows a lateral view of the elbow joint and how it is a composite joint consisting of a hinge or ginglymus joint between the lower end of the humerus and the olecranon of the ulna and the head of the radius.

The superior radial-ulnar joint is considered to be what is called the lateral ginglymus joint.

The articular surfaces are liable to injury.

The various parts of the articular bony surfaces of each joint can severally be involved in different types of injury.

1. The bony structures:

(a) the humerus, involving particularly the lateral epicondyle and capitellar regions, especially in young baseball players

(b)the radial head in stress fractures and osteochondritis

(c) the olecranon process in stress fractures and osteochondritis Fig. 2

Osteochondritis can be seen to affect the articular surfaces of all these bones, particularly those of the radial head and the capitellum and the opposing surfaces of the olecranal and coronoid fossae of the humerus. Also stress fractures can involve the tip of the olecranon fossa of the ulna and the head of the radius. Fig. 3
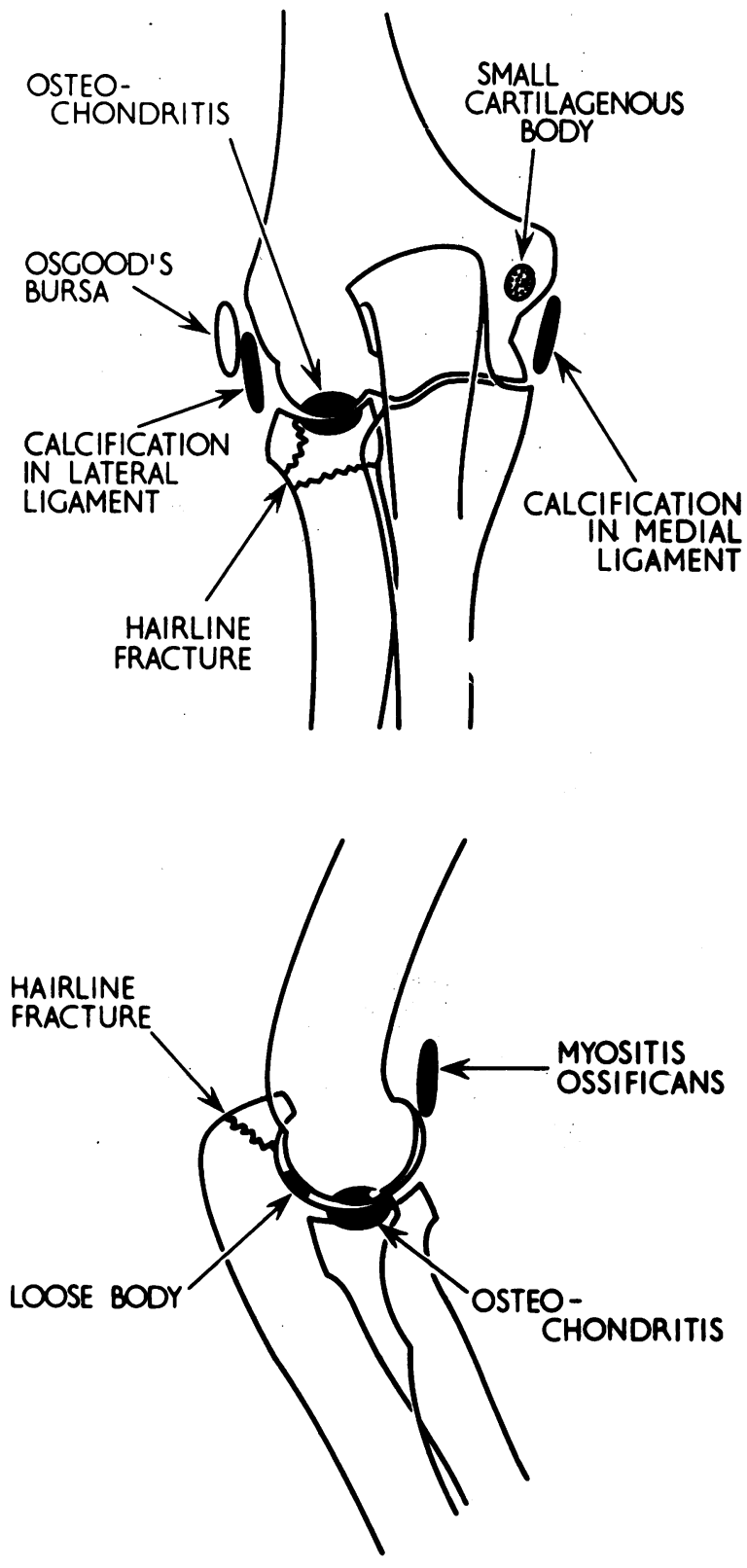

Figs. 2 \& 3 Shows various types of conditions as a result of injury. 
2. Synovial, including the fibrous capsule structures, particularly the lateral and medial collateral, the radiohumeral collateral and orbicular ligaments.

3. Muscles:
(a) the biceps and brachialis in front
(b) the triceps and anconeus at the back
(c) the extensor-supinator group on the lateral side
(d) the flexor-pronator group on the medial side

4. (a) Certain bursae, Osgood's bursa on the lateral aspect if present is involved in certain types of tennis elbow

(b) Occasional bursae on the medial aspect

(c) Olecranon bursa

\section{Nerves:}

The ulna, the median and the radial

6. Blood Vessels:

Both arterial and venous damage is sometimes extensive in dislocations and accounts for complications leading to severe tension in the tissues andVolkmann's Ischaemic Contracture unless the tension is immediately relieved by operation.

Complicated fractures are beyond the scope of this article.

In all cases the active therapeutic approach is applied, as shown on Fig. 4. The surgeon after taking a careful history and making a thorough clinical examination helped by ancillary methods, tries to make as exact diagnosis as possible. Blood tests are taken to rule out an

\section{ACTIVE THERAPEUTIC APPROACH}

\section{FIRST AID TREATMENT}

$$
\begin{aligned}
& \text { MEDICAL CENTRE - } \\
& \text { SURGEON } \\
& \text { EXAMINATION } \\
& \text { DIAGNOSIS } \\
& \text { OPERATIVE } \\
& \text { CONSERVATIVE } \\
& \text { DETAILED INSTRUCTIONS }
\end{aligned}
$$

\section{HOME TREATMENT BY PATIENT}

\section{PHYSIOTHERAPIST}

\section{TRAINER - OCCUPATIONAL THERAPIST - REGIONAL REHABILITATION OFFICER}

Fig. 4 infective, gouty or rheumatic diathesis. Radiographs are taken in several planes and compared with those of the opposite side. The elbow should be treated as a whole arm entity as shown in Fig. 5. In this backhand stroke the hand holding the racquet is assisted by synergist muscles at the wrist, the elbow and the shoulder girdle, but the whole action must take place on a firm, fixed shoulder girdle level.

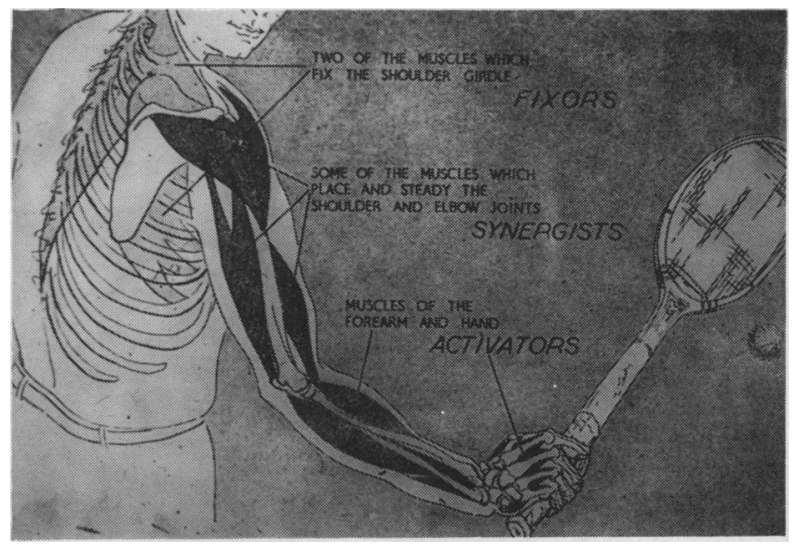

Fig. 5 Shows the principle movement in the arm which must be considered as a whole arm entity. The activators assisted by synergists must work on a prime fixer at the shoulder girdle level.

This is a tennis back hand shot and the hand activators grip the raquet, the synergists which are the wrist, the elbow and shoulder joint must work on a firm prime fixer at the shoulder girdle level.

The influence of poor posture in the prevention of elbow conditions is important. In poor neck and shoulder posture the shoulder girdle is allowed to slump down with the head pushed forward. Fig. 6 and Fig. 7.

There is therefore a tendency for approximation of the clavicle to the structure of the first rib. In such cases as tennis and golf elbow this may be a factor causing compression of the sub-clavicular artery and vein so that there may be not enough arterial blood arriving at the elbow for its requirements, or waste products are not properly eliminated due to pressure on the venous return. If the chin is tucked in and the neck made long and the shoulders elevated and held slightly forwards, there is no compressing on the structures between the clavicle and the first rib. Fig. 8. Even the slight pressure of a tight sleeve may be sufficient to upset the venous return and consequently bring on symptoms.

Relief of tension by aspiration or evacuation by Hilton's method and various forms of physiotherapy are important. Split elbow plasters which can be easily replaced after treatment are applied. A universal sling also gives good support and rest. This sling consists of a loop around the forearm placed 3 " from the point of the elbow, proceeds round the back of the patient to the opposite shoulder, falling down the front of the chest 


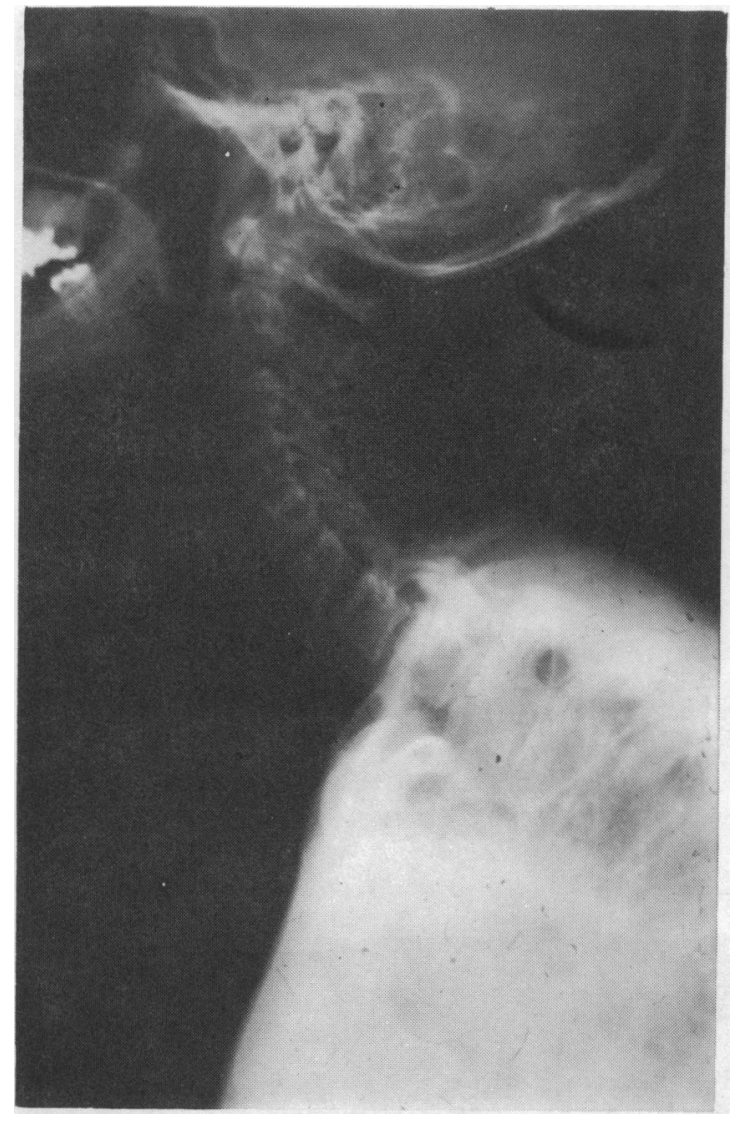

Fig. 6 Shows the head and neck with a marked lordosis as in a slumping posture.

where the wrist is held by a loose clove-hitch which can be shortened or extended. Fig. 9.

Exact instructions as to the right amount of rest and exercise must be laid down and checked to see that they are carried out implicitly. Fig. 10.

The following conditions occur and are now considered:

\section{Dislocations:}

These are divided into those which are stable and those which are unstable. In some cases small flakes of bone are pulled off from the muscle origin from the lateral and medial epicondyles and there is a large effusion in the soft tissues around the elbow. In some cases it is possible to decompress these muscles by making small incisions over the extensor and flexor origins of the muscles, opening up the tissue planes by Hilton's method, i.e. inserting sinus forceps and actually express- ing clot and blood and even small bits of muscular attachment.

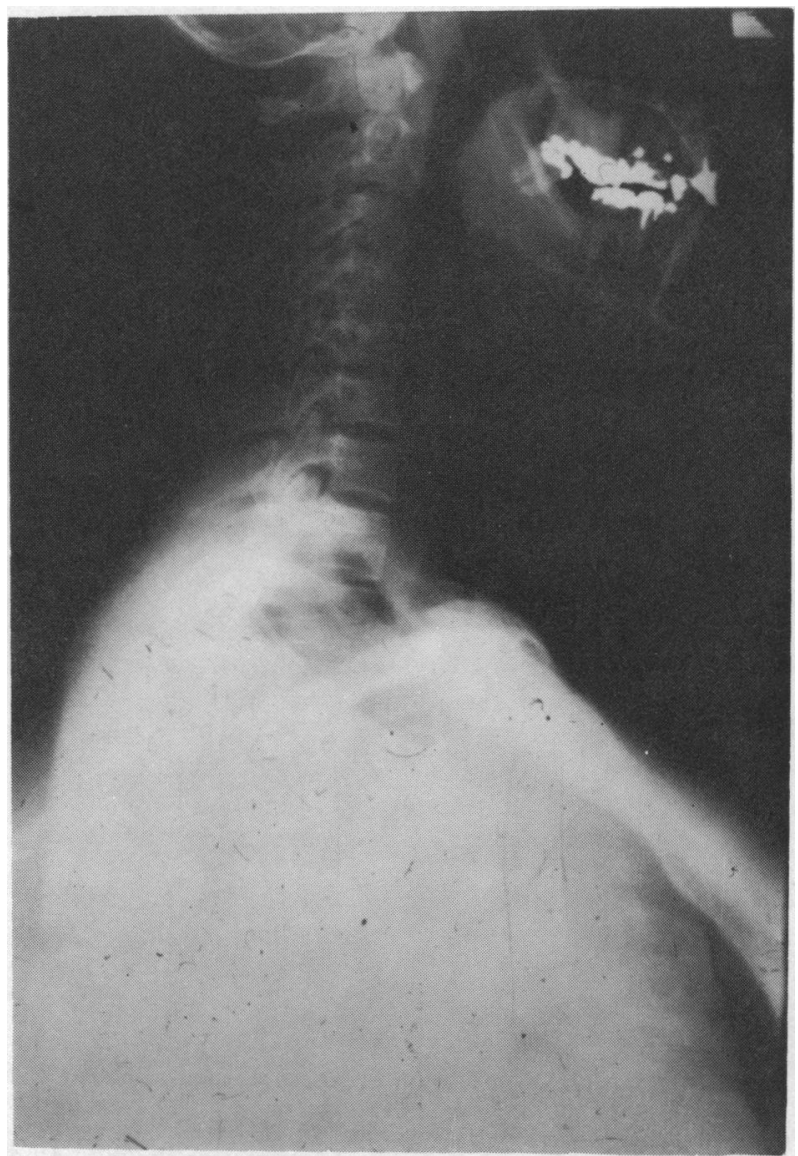

Fig. 7 Shows the head and neck in Active Alerted Posture so that the muscles in front of the anterior cervical muscles are balancing the posterior cervical muscles; each vertebra than becomes one above the other.

In stable fractures the elbow is treated by support in a split plaster which is removed three times a day to allow for hot and cold contrast baths and very gentle active movements which are increased gradually. Reduction of reactionary swelling can be lessened by Faradism and subthermal shortwave.

Unstable dislocations must be kept in a plaster cast without removal for at least three weeks. In certain cases decompression of the elbow structures may be necessary and resuture of ligamentous attachments. During the period of immobilisation in plaster for three weeks very gentle isometric movements are encouraged and the patient is given shortwave through the plaster with gentle faradic contractions of the muscles. 


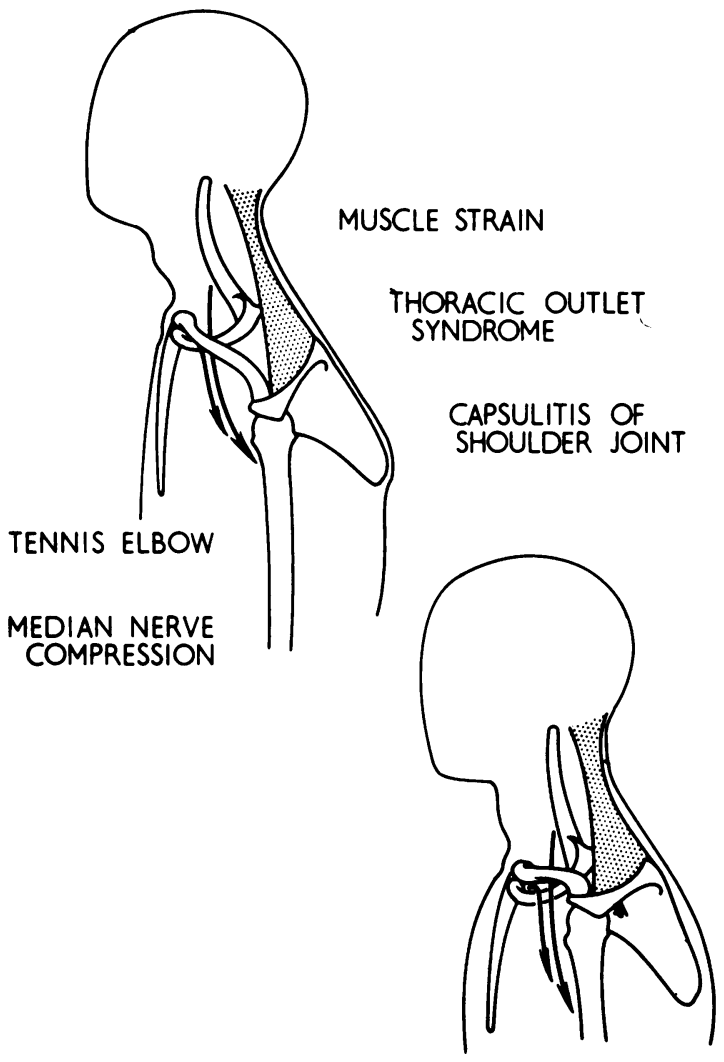

Fig. 8 Shows the difference in the thoracic outlet as a result of Active Alerted Posture compared to slumping posture. The space is wider in Active Alerted Posture as the clavicle is forwards on the first rib therefore giving a greater space for the vital blood vessels and nerves.

\section{The Extensor Supinator Group Injuries}

Strains or tears of the extensor-supinator group includes all cases of so-called "tennis elbow" in which there is a strain or tear of the origin of the extensor muscles. The action is usually combined with bringing the hand from the pronated to the supinated position and therefore the superior radio-ulna joint becomes involved, and the supinator muscles. It is essential for something to be held in the hand, such as a racquet, to produce the strain.

In flexion the biceps brachii is the fixer of the elbow and the supinator of the forearm. In most cases of tennis elbow it will be found that there is a tender point in relation to the long head of biceps at the shoulder joint level in the bicipital groove. Often this becomes very evident with treatment in which not only the elbow should be treated, but the muscles of the shoulder and shoulder girdle.

\section{SALIENT POINTS IN TREATMENT}

\author{
CO-OPERATION OF WHOLE TEAM \\ GENTLENESS STRESSED \\ FORCE NEVER USED \\ MUSCLE SPASM COAXED
}

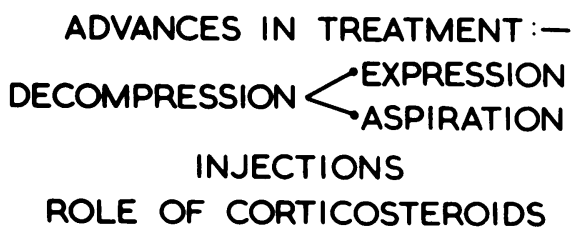

GRADUATED MOVEMENTS

MANIPULATIVE THERAPY

PROGRESSIVE DAILY IMPROVEMENT

Fig. 9 Salient points in treatment.

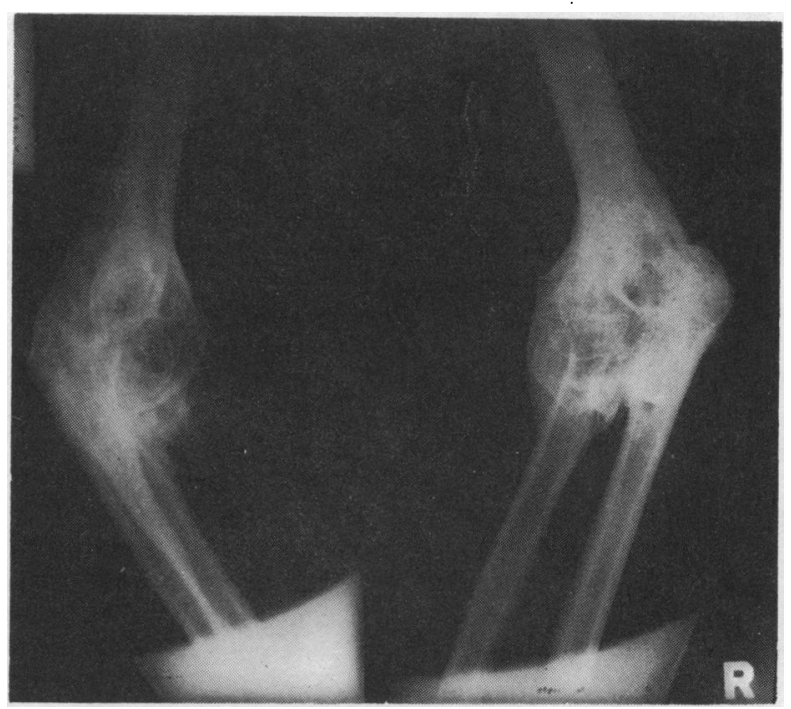

Fig. 10 This shows the result of wrong treatment in a severe elbow injury.

This patient was a well known cricketer and after injury had vigorous massage and exercise by the masseur of an all-in wrestler. The result was complete bony ankylosis.

In some cases in the first degree an actual tear of the tendon or its origin can occur, and if this happens the case should be treated as an acute muscle tear, rested for three weeks, in which xylocaine and hydrocortisone injections with rigorous physiotherapy and support 
should be given and the cases rested from vigorous exercise for three weeks. Most of these cases will clear up in that time. We have devised a special type of sling in which one fixed loop goes around the damaged elbow and the other loop goes back over the good shoulder. A buckle allows for lengthening and shortening of the sling, as already seen in Fig. 9.

On the other hand if this type of case is seen from 6-8 weeks after injury, adhesions have probably formed and often a simple manipulation with or without an anaesthetic will break these adhesions and the case becomes right immediately. The second and third degree type, especially those in which there is an associated chondro-epiphysitis or osteoarthritis are notoriously difficult to get right. However, often if a series of hydrocortisone injections is given at weekly intervals combined with remedial exercises for the whole arm, that is to say fingers, wrist, elbow, shoulder-girdle and neck exercises the condition resolves. Added to this there should be concentrated physical treatment such as ultra-sound, friction massage, gentle manipulation and the majority of them will respond. It would seem that some doctors consider that a simple injection of xylocaine and hydrocortisone is all that is needed to effect an immediate cure. This may be so in many cases, but often several treatments are required and it is essential to treat the whole arm as an entity, because the activator group of muscles must be assisted by the synergic muscles at the wrist, elbow and shoulder and these must all work on the firm prime fixers at the shoulder girdle level (Fig. 3.). The rehabilitation of the patient by exercises at all levels is the most important method of preventing injuries occurring, and when they occur, helping them to become completely right. During the last ten years in eight patients of the over-forty type I have had to carry out the operation described by De Goes (1960). In these cases every type of conservative treatınent has been given before operation, even manipulation under an anaesthetic. In all of them they had a degree of cervical spondylosis, but there was no evidence of high uric acid.

Garden (1961) has suggested elongation of the extensor carpi radialis brevis. Bosworth (1965) has described a removal of the orbicular ligament so as to relieve tension in the elbow joint. De Goes has described a pannus of synovial tissue which grows between the radial head and the capitellum. He calls this the Humeral Meniscus and in the eight cases that I have operated on, I removed this synovial thickening which is seen to pinch between the head of the radius and the capitellum. All these cases have cleared up completely.

Some cases do not respond readily to treatment because the pathological condition is in what I call "the Negative Phase". It is not until the pathological process changes to the Positive Healing phase that recovery starts. There are three degrees of tennis elbow according to the extent to which the tissues are involved.

The first degree is where the musculo-tendinous strain or tear takes place at the origin of the extensor muscles. This often is the type which occurs in the younger athlete between 20 and 30 .

In the second degree the strain is now involving the structures of the superior radio-ulnar joint and there is tenderness over the joint. This is commonly developed in the person who is over 30 .

The third degree is in the older athlete. They have both the tendinous strain at the origin of the extensor muscles and involvement of the superior radio-humeral joint, but superimposed on this there is osteoarthritis appearing in the radio-humeral joint. Of course, if osteochondritis affects the capitellum or radial head, this type of case will give rise to similar symptoms as those of the third degree.

\section{The Flexor Pronator Group Injuries:}

This type gives rise to the golfing elbow and the old type of tennis elbow. In the same way there are three different degrees which seem to affect three different age groups, that is to say between $20 \& 30,30 \& 40$ and over 40 . The treatment of these is similar to that of the supinator-extensor group; i.e. periodic injections of hydrocortisone with the active therapeutic approach to treatment consisting of every form of physiotherapy. Some cases are often slow in recovery and can be associated with small particles of cartilage forming the ligaments, or even giving rise to pressure on the ulnar nerve.

Both the flexor and extensor types of strain can occur so acutely that a period of rest may be necessary for a week before any form of treatment is commenced. These cases are occasionally the ones in which there is a gouty diatheses.

Osteochondritis: can occur in any of the surfaces, for example, of the olecranon surfaces of the ulna, the head of the radius and the corresponding surface of the lateral condyle and capitellum. Small loose bodies can become separated into the joint, cause locking, and have to be removed. Sometimes on radiographs loose bodies appear to be present in the joint, but in fact locking does not occur, because they are attached to the capsule of the joint and are not free. Therefore operative removal should be carried out with caution.

Myositis Ossificans: There is no question that this condition can be accelerated by too vigorous after treatment of a dislocation and injuries to the elbow. The 
common place for myositis ossificans of the muscle is in the tendons of the biceps and the brachialis in front of the joint. Calcification of the ligaments can take place, however, and must be distinguished from the actual myositis in the muscles.

Hairline stress fractures: These occur in the radial head and lip of the olecranon.

Spur Formation: This can happen as a periostitis forming in the origin of the muscles particularly on the lateral aspect, and can be associated with tennis elbow.

Anticubital swellings: In baseball players, this occurs in the region of the medial epicondyle and the flexor origin, and extands forwards. It can be associated with bursal formation in this area, and neuritis affecting the median nerve. In some cases operative interference is indicated.

Small ossicles occasionally form in the actual ligaments themselves some way from the origin and can give rise to recurring pain and disability and have to be removed. These are common in the medial collateral ligament.

Pull-pushed elbows: Occasionally this entity occurs as a result of a fall on the outstretched hand where the head of the humerus is pushed up through the orbicular ligament. It has been recorded that rarely the forearm has been severely wrenched and the radial head has been pulled down through the orbicular ligament. Treatment consistes of either pushing or pulling these so as to reduce the subluxation. The elbow must be treated as a joint sprain following this.

Nerve involvement: Any athletic injury causing contusion of the tissues can promote increased fibrosis of the tissues and increase the chance of entrapment neuropathy. Kopell and Thompson (1963) describe in their book many cases of tennis elbow which are caused by the entrapment of the superficial branch of the radial nerve as described above. We find that often in certain cases this is only partially so and is associated with the involvement of the outer side of the elbow structures as in third degree and unless these lesions of the extensorsupinator group are treated also the individual with tennis elbow does not improve. Often treatment directed to freeing this pressure by injections and physiotherapy will clear up this entrapment syndrome also.

Entrapment neuropathy of the median nerve at the higher level is caused by the nerve going through a

\section{Acknowledgements:}

Fig. 5 Is from Tucker W. E., "Home Treatment and Posture": Livingstone 1969. tunnel under what is called the ligament of Struthers which runs from the supro-condylar process down to the medial epicondyle. At the lower level it is entrapped between the heads of the pronator teres muscle as it goes down to lie on the substance of the flexor digitorum sublimus.

Ulnar nerve entrapment occurs when it passes behind the medial epicondyle due to fascial thickenings and entrapment also may take place as it passes between the humeral and ulnar heads of the flexor carpi ulnaris.

The radial nerve can be entrapped, particularly the superficial branch, by the fascial edge of the origin of the extensor carpi radialis brevis. The deep branch and the recurrent branch can be involved either in this sheet of fascia or as it passes between the two parts of the supinator brevis.

If there is evidence of muscular involvement or nerve entrapment, transplant in front of the elbow is essential for quicker recovery. If there are minor sensory symptoms these are sometimes relieved by injections and physiotherapy.

\section{Summary:}

1) Tennis injuries are common and involve the extensor supinator group of muscles on the lateral side of the joint. In the older athlete the radio-humeral and superior radio-ulnar joints become involved also. Muscles of the shoulder joint and shoulder girdle are often secondarily affected, especially the long head of biceps in the bicipital groove.

2) The golfing elbow affects the medial flexor pronator group. This type of strain used to be common in tennis at the beginning of the century when the forehand drive was executed with the head of the racquet down and top spin was imparted to the ball. Golfers often suffer from a typical tennis elbow of the left elbow due to extending and supinating the forearm on the flexed elbow in following through their stroke.

Cricket, baseball, pitching and javelin-throwing cause strain on the flexor pronator group if the action is carried out with the round arm method. If the throwing is executed with the arm elevated above the shoulder and then the elbow forcibly extended, stress fractures of the lip of the olecranon can occur. In young baseball players separation of the lateral epicondyle and capitellum as an osteochondritic lesion is not uncommon.

Fig. 9 Is from Tucker W. E., "Home Treatment in Injury and Arthritis": Livingstone 1961.

Gratitude is expressed to the publishers for permission to reproduce these figures. 
References:

1. DE GOES, H., (1960) "The Radio-humeral meniscus and its relation with tennis elbow". Archives of Interamerican Rheumatology, 111:4 December, 1960.

2. BOSWORTH, D. M., “Surgical Treatment of Tennis Elbow”. Journ. Bone Joint Surg. 47A:December, 1965.

3. GARDEN, R. S., (1961) “Tennis Elbow”. Journ. Bone Joint Surg. 43:100.

4. KOPELL, H., and THOMPSON, W. "Peripheral Entrapment Neuropathices", p. 125. The Williams and Wilkins Co., Baltimore 1963.

\section{INJURIES TO THE UPPER LIMB IN JUDO}

PHYLLIS ELLIOTT, M.B., Ch.B.

Physiology Dept. Sheffield University.

Hon. Medical Officer, British Judo Association.

There are a variety of ways in which injuries to the upper limb may be sustained in Judo, and they may affect either the person who is applying a technique (Tori) or the person on whom the technique is applied (Uke).

Considering Uke first, the causes of injury to the upper limb fall into two main categories - armlocks and throws. An armlock is a technique applied to the elbow, with the arm in either a bent or a straight position, which imposes strain on the ligaments and would, if continued, cause dislocation; Uke therefore submits and Tori gains a point in contest. However, occasionally in contest, the lock may be applied over-enthusiastically by Tori, or Uke may be a little slow in submitting due to attempting first to escape from the lock. In these cases, especially in the case of a bent armlock, the medial ligament of the elbow may be damaged. In Judo, the elbow is the only joint to which locks may be applied; Knee-locks used to be allowed but were banned several years ago, presumably because they were too liable to cause injury. However, while fighting, other joints may be accidentally injured - for example, fingers or wrists due to the hand becoming trapped in the opponent's jacket - or a shoulder may be subjected to strain by a badly applied elbow-lock.

In the case of throws, injury may be caused either by landing on the point of the shoulder, when Uke has not been turned sufficiently to cause him to land on his back, or by a fall on the outstretched hand if for some reason Uke puts an arm out straight as he is being thrown. Landings on the point of the shoulder produce acromio-clavicular lesions (sufficiently frequent that this is called "black-belt shoulder" by the competitors!), fractured clavicles and occasionally sterno-clavicular lesions. The fall on either an outstretched hand, or sometimes the elbow, can of course produce many different injuries; the one. I have seen most frequently is dislocation of the elbow, plus the occasional dislocated shoulder.

The person applying the techniques is of course at less risk, but with some throws, he may also land on the point of his shoulder while throwing his opponent. His other major source of possible injury is again trapping of the hand in the opponent's jacket while throwing him, or during groundwork. Sometimes the wrist will be subjected to considerable strain if he allows it to become hyper-extended while throwing his opponent, instead of keeping the hand in line with the fore-arm.

All of the above may make Judo sound a very dangerous sport; however, a large proportion of people practising Judo do so for many years without suffering more than the occasional bruise. The above observations have been gleaned over 14 years in Judo, both as a participant and as Medical Officer at many of the major Judo events. 